\title{
Cervical laminoplasty
}

Published online: 4 February 2010

(C) Springer-Verlag 2010

\section{Keith D. Luk MD, Vijay Kamath MD, Ashwin Avadhani MD and S. Rajasekaran MD}

\author{
Keywords \\ Double door laminoplasty · Spondylotic myelopathy · \\ T-saw
}

\section{Introduction}

Posterior spinal decompression via expansive laminoplasty is designed to preserve the posterior structures of the cervical spine, so as to prevent postoperative development of instability and cervical kyphosis. The T-saw laminoplasty is a double door laminoplasty using a thin thread saw of $0.54 \mathrm{~mm}$ in diameter designed by Katsuro Tomita. A specially designed polyethylene outer sleeve $(1.2 \mathrm{~mm}$ in diameter) is used to guide the passage of the saw under the laminae.

Splitting the spinous process using the $T$ saw laminoplasty provides adequate spinal cord decompression and has the advantages of decreased blood loss and operative time, less bone loss, facilitation of posterior arch reconstruction, and decreased risk of dura and spinal cord injury.

This is an illustrative case of a multilevel spondylotic stenosis causing myelopathy managed with a double door laminoplasty performed using the T-saw.

Electronic supplementary material The online version of this article (doi:10.1007/s00586-010-1314-0) contains supplementary material, which is available to authorized users.

\section{Case description}

The patient is a 65 year old male with progressive numbness in all four limbs, loss of manual dexterity and unsteady gait of 3 months duration. He had clinical features of cervical myelopathy. The radiographs showed normal cervical lordosis with a reduced disc space height and an anterior osteophyte at C5-6. The MRI showed multilevel cord compression from C3-4 to C6-7. The compression was from both anterior and posterior due to degenerated central disc protrusions, and ligamentum flavum hypertrophy respectively. Cord signal intensity changes were seen at the C5-6 level. In view of the multilevel compression it was decided to perform an expansive laminoplasty.

\section{Surgical procedure}

The procedure is performed under general anesthesia. Neck hyperextension during intubation should be avoided as this may cause further canal compromise and precipitate or exacerbate neurological deficits. The patient was positioned prone with the head in a Mayfield head rest or clamp and care taken not to position the neck in kyphosis.

A posterior midline exposure is performed from $\mathrm{C} 2$ to $\mathrm{C} 7$, avoiding detachment of the semispinalis muscles from the $\mathrm{C} 2$ spinous process. This maintains effective cervical extensor power and aids in maintaining cervical alignment. The supraspinous ligament attachment to $\mathrm{C} 7$ should also be maintained. Subperiosteal exposure of the posterior elements was performed up to the medial portion of the lateral mass, so that the bone troughs can be made at the laminafacet junction. Injury to the facet capsules should be avoided. 
Decompression is from C3 to C6. The ligamentum flavum was excised in the midline to expose the epidural space at C2-3 and C6-7. The polyethylene sleeve was passed rostrally from $\mathrm{C} 6-7$ level along the midline epidural space and the tip retrieved as it appeared in the flavectomy at $\mathrm{C} 2-\mathrm{C} 3$. The saw was advanced through the sleeve, and then the sleeve withdrawn over the saw. Each end of the T saw is grasped with a special clamp.

After ensuring adequate cervical lordosis (by anteriorly directed pressure on the $\mathrm{C} 4$ spinous process), the $\mathrm{T}$-saw is pulled tight to fit snugly to the inner wall of the laminar arch just at the midline. Continuous reciprocating motion was used to cut the spinous processes from ventral to dorsal, away from the dura and spinal cord. The direction of the saw should be adjusted to ensure a central split of the spinous processes. The ligamentum flavum, supra- and interspinous ligaments are automatically divided at the midline as the saw cut advanced.

Bilateral troughs are then made in the lamina-facet junction with a high-speed $3 \mathrm{~mm}$ diameter burr. Care is taken to thin the inner cortex only to the point of plastic deformability. The split laminae are opened in sequence-like French doors along the decompression zone, aided by the use of a modified laminar spreader. Dural expansion and pulsation usually are observed once the hemilaminae are separated by 15-20 mm or approximately 40-45 degrees. Careful dissection of any epidural adhesions facilitates the decompression. The decompression typically extends from C3 through C6, but undercutting of the C7 lamina should be performed in the presence of compression at C6-7 level. If $\mathrm{C} 2$ decompression is required, it usually can be undercut (dome laminoplasty) using a diamond burr, without compromising its structural integrity or detaching the extensors.

Separation of the hemilaminae is maintained by iliac crest autografts, which are held in place with non-absorbable sutures passed through holes of the halved spinous processes fashioned with a 1-millimeter burr.

\section{Post-op procedure}

The patient is allowed to ambulate on the second postoperative day with a soft cervical collar for pain relief and comfort. The collar is used for 4-6 weeks.
Postoperative radiographs are assessed for the maintenance of separation of hemi-laminae by the graft/spacer. Adequacy of decompression may be assessed by an MRI scan or a CT myelogram if required.

\section{Discussion and conclusion}

Posterior spinal decompression via expansive laminoplasty is designed to preserve the posterior structures of the cervical spine, so as to prevent postoperative development of instability and cervical kyphosis.

A laminoplasty is indicated in cervical spinal canal stenosis due to developmental, multilevel spondylotic or OPLL etiology in patients with a lordotic cervical spine. The procedure is contraindicated in the presence of pre operative cervical kyphosis, in the presence of ossification of the ligamentum flavum or epidural scar from previous posterior cervical procedures. Sublaminar passage of the polyethylene sleeve would either be unsuccessful or dangerous in such circumstances.

Separation of the hemilaminae may be maintained by corticocancellous iliac crest or rib autografts or specially designed ceramic/hydroxyapatite spacers or plates. Grafts or spacers are held in place with sutures or wire that are passed through holes in the halves of the lamina.

\section{References}

1. Tomita K, Kawahara N, Toribatake Y, Heller JG (1998) Expansive midline T-saw laminoplasty (modified spinous process-splitting) for the management of cervical myelopathy. Spine 23:32-37

2. Edwards CC 2nd, Heller JG, Silcox DH 3rd (2000) T-Saw laminoplasty for the management of cervical spondylotic myelopathy: clinical and radiographic outcome. Spine 25:1788-1794

3. Martin-Benlloch JA, Maruenda-Paulino JI, Barra-Pla A, LaguiaGarzaran M (2003) Expansive laminoplasty as a method for managing cervical multilevel spondylotic myelopathy. Spine 28:680-684 